\title{
Interferometry and Laser Control with Solid Fabry-Perot Etalons
}

\author{
Don G. Peterson and Amnon Yariv
}

\begin{abstract}
The use and analysis of solid Fabry-Perot etalons for interferometry and laser control are discussed and supported with experimental data. Low angle scattering is found to be an important factor influencing finesse and peak transmission. Thermal tuning sensitivity and wedge-angle control with thermal gradients are analyzed and illustrated. Control of laser oscillations using a solid-state etalon as a laser cavity end mirror is discussed. The use of the solid etalon as an optical cavity coupler is applied to the problem of sideband energy removal from an internally modulated laser.
\end{abstract}

\section{Introduction}

The Fabry-Perot etalon is used widely in high resolution optical spectroscopy. The plane parallel reflectors which are used to form the etalon can be made flat to a high degree, and when coated with multilayer dielectric coatings can have very high reflectance $(>99 \%)$ with very low losses. If we consider the etalon as an electromagnetic resonator, its quality factor $Q$ is extremely high (typically $>10^{7}$ ), which fact explains its high spectral resolution.

The physical factors causing departure from ideal ${ }^{1}$ performance have been the subject of several investigations. $^{1-4}$

We have studied experimentally Fabry-Perot etalons made by depositing multidielectric layers on opposite surfaces of optical quartz flats, thus forming solid-state etalons hereafter designated as SSE's. In this case the resonance properties of the etalon depend on the optical properties of the quartz. This gives rise to a number of new effects and potential applications, some of which will be described in this paper.

\section{Preparation of the Solid Etalons}

The optical flats were made from schlieren-grade fused quartz. The plane surfaces were ground flat to $\lambda / 100$ in the visible and were made parallel with a residual wedge angle of 3-4 sec. Standard spacings of $0.6 \mathrm{~cm}$ and $1.1 \mathrm{~cm}$ were used. The surfaces were finally coated with multilayer dielectric mirrors with $90 \%$ reflectance at $6328 \AA$.

Don G. Peterson is with the Lockheed Palo Alto Research Laboratory, Palo Alto, California 94304; Amnon Yariv is in the Division of Engineering and Applied Science, California Institute of Technology, Pasadena, California 91109.

Received 21 December 1965.

This work was supported by the Lockheed Independent Research Fund.

\section{Etalon Parallelism Testing}

To test the parallelism, we illuminated the etalon with a well-collimated laser beam at $6328 \AA$ at near normal incidence. Under these conditions, and for perfectly parallel reflectors, the whole surface of the output end of the etalon should transmit the laser beam whenever the etalon length is equal to an integral number $m$ of halfwavelengths in the quartz, that is, when

$$
2 L=m \lambda_{0} / n,
$$

where $L$ is the spacing between the reflectors, $m$ is the interference order, $\lambda_{0}$ is the input wavelength in a vacuum, and $n$ is the index of refraction of the etalon material. If the reflectors are not perfectly parallel, only those portions of the surface for which Eq. (1) is satisfied (not necessarily with the same integer $m$ ) will transmit. Each transmitting portion of the etalon corresponds to a contour of constant optical spacing. For a monotonic variation in spacing, adjacent fringes correspond to a variation in spacing equal to half a wavelength. No more than one fringe could be seen at any one time over the 1-cm-diam output face of the etalon. Two fringes separated by $1 \mathrm{~cm}$ would correspond to a wedge angle of 5 sec of arc. On that basis, the deviation from parallelism was estimated as $\sim 3$ sec. A photograph of the transmitted light pattern at the output surface of the etalon is shown as Fig. 1. The existence of a small wedge angle can be utilized in tilted-plate interferometry ${ }^{1}$ as demonstrated recently by Moos et al. ${ }^{5}$ This application is based on the fact that, in a given order $m$, different frequencies present at the input will be transmitted through different locations in the etalon. Each frequency seeks out the locus of constant separation which, according to Eq. (1), is appropriate to it. If the variation of separation across the output surface is known, the spectral profile of the input light can easily be reconstructed. To avoid overlap between adjacent orders, $\Delta|m|=1$, the bandwidth of the input light 
Fig. 1. Photograph of the transmitted light pattern at the output surface of a solid-state etalon when illuminated with a well-collimated laser beam ( 6328 A) at near normal incidence. The single fringe indicates a wedge angle of approximately $3 \mathrm{sec}$ of arc.

must be smaller than half the spectral range $c / 2 n L$ of the etalon. The spectral range in our experiment was $\sim 18$ $\mathrm{Gc} / \mathrm{sec}$.

\section{Study of Thermal Transients}

One interesting application of the SSE is in the study of thermal transients. The experimental arrangement is shown in Fig. 2. A temperature step was applied to the outer surface of the etalon by suddenly increasing the temperature of the water flowing in the heat exchanger surrounding the SSE. A series of photographs of the output surface of the etalon are shown in Fig. 3. Beginning with Fig. 3(a), these pictures were taken in an increasing-time sequence subsequent to the application of the temperature step. Figure 3(a) shows the straight fringe caused by the initial wedge angle between the plane parallel surfaces as described in Secs. II and III. The radial temperature gradient which is caused by the heat step is illustrated in Figs. 3(b), 3(c), and 3(d). The difference in thermal expansion between the outer (cylindrical) surface of the etalon and the etalon axis causes the input and output surfaces of the etalon to be slightly curved. (In addition the index of refraction also increases with temperature thus causing an increase in optical path length $n L$. In fact this effect is an order of magnitude larger than thermal expansion as is discussed in Sec. VI.) If the surfaces are plane parallel at the beginning of the step, the equal separation contours constitute a family of concentric circles. The small deviation $(\sim 3 \mathrm{sec})$ from plane parallelism that exists causes a distortion of these circles. At the height of the transient, Fig. 3(c), the resulting sphericity of the surfaces is large enough to dominate over the residual wedge angle and consequently the three fringes are nearly circular. As discussed in Sec. VII, the tempera-

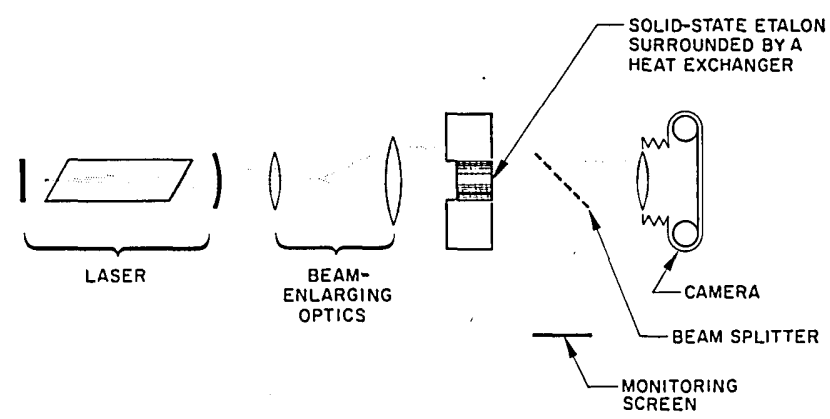

Fig. 2. Diagram of the experimental set up for studying thermal transients in solid state etalons. ture difference per fringe is, in this case, $2.5^{\circ} \mathrm{C}$, so that, at the instant at which Fig. 3(c) was photographed, there existed a $9^{\circ} \mathrm{C}$ temperature difference between the outside surface of the etalon and its center. The approach to a steady state at the elevated temperature and the return to the condition of plane surfaces is shown in Figs. $3(\mathrm{~d})-3(\mathrm{~g})$.

Figure 3 illustrates the use of the SSE for the study of thermal transients. It must be added that the temperature difference between two adjacent fringes is \pm 2.5 ${ }^{\circ} \mathrm{C}$, or $0^{\circ} \mathrm{C}$, so that a unique assignment of temperatures is possible only when the temperature transient is a monotonic function of time. This is the case in our experiment so that we can obtain information about the temporal and radial variation of temperature, $T(r, t)$, in the etalon material.

The substitution of a high-speed motion picture camera will make it possible to apply this method for photographing high-speed thermal transients in transparent solids, such as, for example, the study of thermal transients in solid laser rods during the pumping pulse.

\section{Solid-State Etalon as an Optical Transmission Resonator}

The transmission of a lossless plane parallel FabryPerot etalon of length $L$, index of refraction $n$, and mirror reflectivity $R$ is given by

$$
\tau=\frac{I_{\text {trans. }}}{I_{\text {incid. }}}=\frac{1}{1+\left[4 R /(1-R)^{2}\right] \sin ^{2}\left(2 \pi n L \cos \theta / \lambda_{0}\right)},
$$

where $\lambda_{0}$ is the free-space wavelength of the incident radiation and $\theta$ is the angle between the incidence direction and the normal to reflecting surfaces. The resolution of the etalon can be expressed in terms of the full width at half-maximum $(\Delta \nu)_{\frac{1}{2}}$ of the transmission curve $\tau$ vs $\nu$. When $R$ is near unity, Eq. (2) yields

$$
(\Delta v)_{\frac{1}{2}}=c_{0}(1-R) / 2 \pi n L R^{\frac{1}{3}},
$$

where $c$ is the velocity of light in vacuum. An alternative way of describing the resolution is in terms of the finesse $f$, which is the ratio of the frequency separation between successive transmission peaks $c / 2 n L$ to $(\Delta \nu)_{\frac{1}{2}}$. Using Eq. (3) gives

$$
f=\pi R^{\frac{1}{2}} /(1-R) .
$$

To determine the finesse and peak transmission of the SSE, we measured its $\tau$-vs- $\nu$ characteristics. This was done by letting a collimated single-mode laser beam at $6328 \AA$ impinge normally on the etalon. The transmitted beam was incident on a photomultiplier and the output current applied to a strip-chart recorder. The optical length of the etalon was swept by varying the temperature of the water flowing through the heat exchanger. The resulting transmission curve is shown in Fig. 4.

The peak transmission is $83 \%$ while the finesse is measured as $f=26.5 \pm 2$. These results are discussed in the following section. 


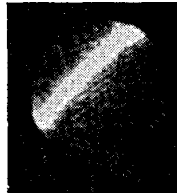

(a)

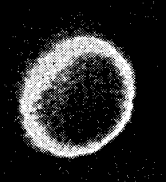

(b)

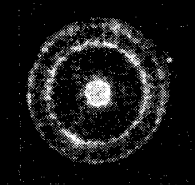

(c)

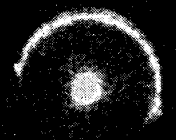

(d)

\section{.}

(e)

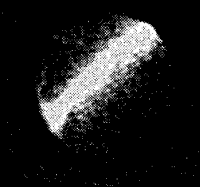

(g)

Fig. 3. Transient transmission fringe patterns caused by a thermal step applied to cylindrical surface of a solid-state etalon. (a) The residual wedge angle fringe before application of thermal step. (b) The wedge angle begins to curve shortly after application of step. (c) The wedge angle fringe has been almost completely overcome by the transient. The concentric fringes of several orders are centered very near the center of the SSE. (d) and (e) The transient fringes decay. (f) All fringes disappear. (g) A new steady state at a higher temperature has been reached, and a single straight fringe due to the residual wedge angle is again seen.

\section{Low Angle Scattering and Its Effect on the Etalon Performance}

According to Eq. (2) the peak transmission of a lossless etalon is unity. The finesse, according to Eq. (4) and the mirror reflectivity $R=0.9$, should be $f=30$. The measured values, on the other hand, are $\tau_{\max }=$ $0.83, f=26.5 \pm 2$. The discrepancy is most likely due to the existence of low angle light scattering in the etalon. This scattering can exist either in the bulk material or in the dielectric mirrors. In the limit of high reflectivity and small losses, both loss mechanisms lead to the same degradation of finesse and peak transmission and, consequently, cannot be separated in a measurement on a single etalon.

We characterize the intensity attenuation per pass by $e^{-\sigma}$ (so that, for $\sigma \ll 1, \sigma$ is the fractional intensity loss per pass) and take the fractional scattering loss per reflector as $\gamma$. We now have $R^{\prime}+T+\gamma=1$, where $R^{\prime}$ is the specular reflectivity per reflector and $T$ its transmissivity. We can view this relation as a definition of the reflector scattering loss $\gamma$. Rederiving the transmission equation of the Fabry-Perot etalon under these conditions leads to

$\frac{I_{\text {trans }}}{I_{\text {incid }}}=\left(\frac{1-R^{\prime}-\gamma}{1-R^{\prime} e^{-\sigma}}\right)^{2} \frac{1}{1-\left[4 R^{\prime} e^{-\sigma} /\left(1-R^{\prime} e^{-\sigma}\right)^{2}\right] \sin ^{2}(\delta / 2)}$,

where $\delta=4 \pi n L \cos \theta / \lambda_{0}$.

In our work $R^{\prime}$ is not known, since the angular resolution of the equipment used for reflectivity measurements cannot separate the low-angle scattering loss $\gamma$ from $R^{\prime}$. The reflectivity thus obtained is the sum of both and is taken as $R=R^{\prime}+\gamma$. When the conditions $\gamma \ll 1$ and $\sigma \ll 1$ are satisfied, which is the case in our experiment, we obtain from Eq. (5) the following expressions in which only $R$, rather than $R^{\prime}$, appears

$$
\begin{aligned}
& \tau_{\max }=1-\{(\gamma+R \sigma) /[(1-R)+(\gamma+R \sigma)]\}, \\
& f=\pi[R-(\gamma+R \sigma)]^{\frac{1}{2}} /[(1-R)+(\gamma+R \sigma)],
\end{aligned}
$$

so that from the finesse $(f)$ and peak transmission $\left(\tau_{\max }\right)$ data we can calculate the loss factor $(\gamma+R \sigma)$. Using the experimental values $\tau_{\max }=0.83$ and $R=0.9$ in Eq. (6a) gives $0.9 \sigma+\gamma=0.98 \%$. As a check, we substitute this value in Eq. (6b) and get $f=27.0$, which is to be compared with the experimental value of $f=2.65 \pm$ 2.
It is clear from the above data and discussion that by measuring the peak transmission of the etalon we have an extremely sensitive tool for measuring small amounts of optical losses. In our case a $0.98 \%$ loss per pass causes a degradation in peak transmission from 1.0 to 0.83. By increasing the reflectivity $R^{\prime}$, we should be able to detect losses of less than $10^{-4}$ per pass. Residual low angle scattering in the bulk or in high quality reflectors is the limiting factor in the performance of many optical systems using coherent light, so that their detection and characterization are a problem of growing concern.

As mentioned earlier in this section, we were not able in this experiment to separate the losses in the mirrors from those in the bulk. In view of the high grade optical material used, it is reasonable to assume that the $0.98 \%$ loss per pass is due to low angle scattering in the multidielectric mirrors rather than in the quartz. This problem can be settled by noting the dependence of $\tau_{\max }$ (or $f$ ) on the length $L$ of the etalon. If the losses were completely due to the mirrors, $\tau_{\max }$ and $f$ [according to Eq. (6a)], would be independent of $L$. If, on the other hand, the losses were distributed uniformly in the bulk, an increase in $L$ would cause a degradation of both $\tau_{\max }$ and $f$. Experiments on etalons with different lengths are contemplated.

A visual demonstration of the low angle scattering was provided by focusing the output beam emerging from the etalon using a long focal length lens. A photograph of the focused beam is shown in Fig. 5 . The low angle scattering feeds light into lower-order off-axis Fabry-Perot modes which give rise, when focused, to the characteristic Fabry-Perot family of concentric circles. From the number of such circles

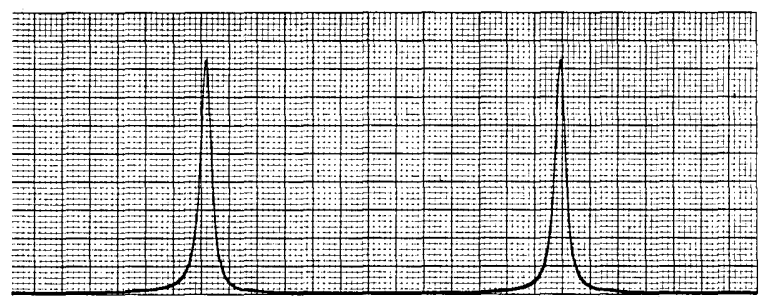

Fig. 4. The transmission characteristics ( $\tau$ Vs $\nu$ ) of a solid state etalon. The full vertical scale corresponds to $100 \%$ transmission. The intermode spacing on the horizontal scale is approximately $18 \mathrm{Gc} / \mathrm{sec}$. 


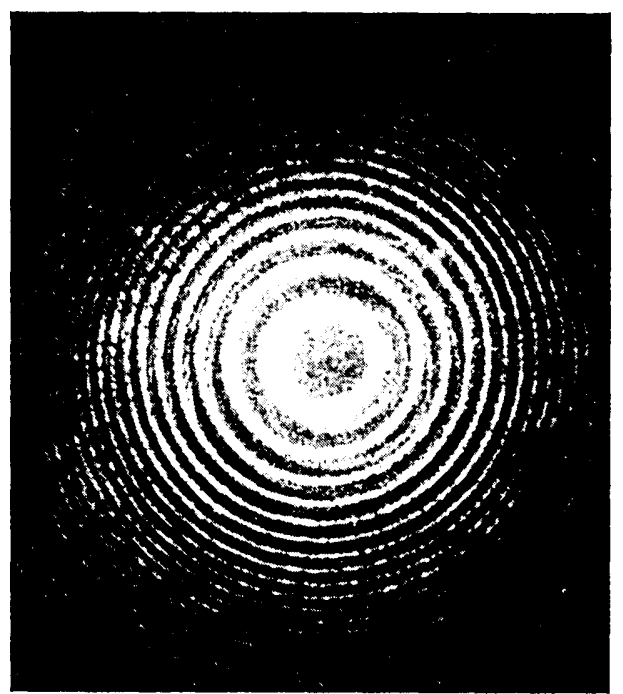

Fig. 5. Distribution of intensity in the far field of a solid state etalon obtained by masking the central spot and focusing the scattered energy at the output on the film plane. The first ten rings correspond to scattering into a half cone-angle of approximately $1^{\circ}$.

that are excited appreciably $(\sim 10)$, we estimate the effective cone angle of the low-angle scattering as $\theta$ $\approx 1^{\circ}$.

\section{Thermal Tuning}

It follows from Eq. (2) that a change in length of $\delta L$ $=\lambda_{0} / 2$ causes the passbands of a Fabry-Perot etalon to tune over the full spectral range of the etalon $\Delta \nu=$ $c / 2 n L$. A fine control of a Fabry-Perot etalon operating in the visible thus requires controlling $L$ to within a small fraction of a micron. This control can easily be achieved by varying the etalon temperature $T$. This point becomes evident if we consider the dependence of the mode number $m$ (the number of half-wavelengths contained in $L$ ) on temperature. From Eq. (1) we have

$$
d(m)=\frac{2 n L}{\lambda_{0}}\left(\frac{1}{L} \frac{d L}{d T}+\frac{1 d n}{n d T}\right) d T .
$$

The first term in the parenthesis on the right side of Eq. (7) is the thermal expansion coefficient of the material while the second term allows for the temperature dependence of the index of refraction $n$. Tabulated values for these coefficients are ${ }^{6}$

$$
\begin{gathered}
\frac{1}{L} \frac{d L}{d T}=\frac{0.6 \times 10^{-6}}{{ }^{\circ} \mathrm{C}}, \\
\frac{1 d n}{n d T}=\frac{7 \times 10^{-6}}{{ }^{\circ} \mathrm{C}} .
\end{gathered}
$$

Substituting these values in Eq. (7) and using the constants $n=1.457, L=1.1 \mathrm{~cm}$, and $\lambda_{0}=6328 \AA$ that are appropriate to our experiment yields

$$
d m / d T=0.41 \text { fringe } /{ }^{\circ} \mathrm{C},
$$

so that approximately a $2.5^{\circ} \mathrm{C}$ change in temperature sweeps the etalon over its complete spectral range. To check this number we performed an experiment similar to that leading to Fig. 4 . The water temperature was increased by $33^{\circ} \mathrm{C}$, and the number $\Delta m$ of transmission peaks swept by the etalon was monitored. The result was $\Delta m=11 \pm 2$, while the number predicted by Eq. (8) is 12.4. The uncertainty of \pm 2 is attributed to our lack of absolute temperature control which made the starting point and the final point of the sweep uncertain by one fringe each. It is interesting to note that this procedure affords an easy means for measuring the sum of the expansion and index temperature coefficients, and secondly, that by controlling the temperature of a SSE etalon to within a fraction (for instance, 0.1 ) of a degree, we can stabilize its spectral characteristics by an amount which exceeds its resolution.

\section{Thermal Control of the Wedge Angle}

In the previous section, we have seen how thermal gradients change the effective mirror separations. It appears that properly applied temperature gradients could be employed to overcome and perhaps control the residual wedge angle. In an attempt to do this, we modified our heat-exchange mount from a single to a double chamber mount so that hot water could be passed through one side and cold water through the other without mixing. A picture of the heat-exchange mount is shown in Fig. 6, and a cutaway view is shown in Fig. 7. The SSE was rotated within the mount so that a temperature gradient could be maintained parallel to the plane containing the wedge angle and perpendicular to the SSE symmetry axis. Figure 8 shows the results. The first photograph [Fig. 8(a)] shows how the transmitted interference pattern appeared before any gradient was applied and corresponds to the residual wedge angle of $\sim 4$ arc sec. Figure $8(\mathrm{~b})$ shows four fringes resulting from about $12{ }^{\circ} \mathrm{C}$ temperature difference applied across the SSE in a direction which causes

Fig. 6. Photograph of double chamber heat-exchanging mount used to apply ther. mal gradients to solid state etalons for wedge angle control.

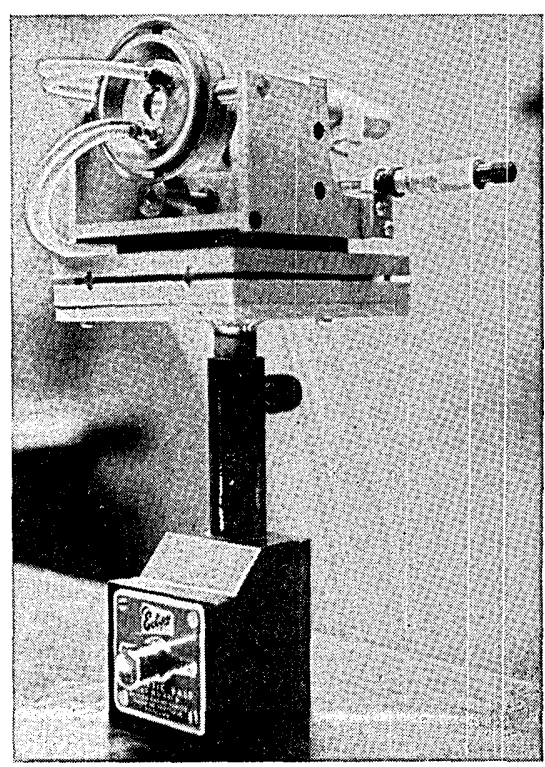




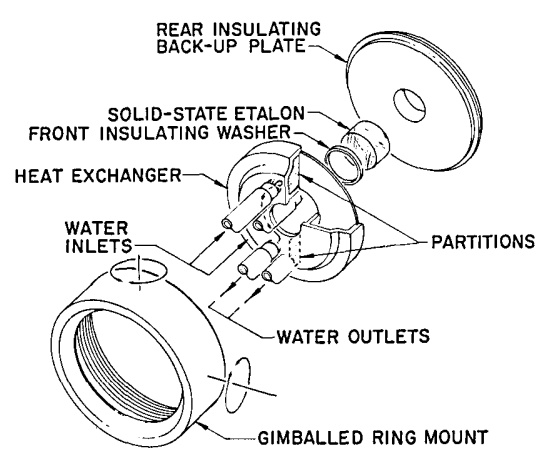

Fig. 7. Cutaway view of the double chamber heat-exchanging mount used in wedge angle control experiment.

the wedge angle to increase. Figure $8(\mathrm{c})$ shows the same fringe pattern again, 1 min later. A small shift downward indicates that the average temperature of the SSE has changed slightly. The hot and cold water inputs were then interchanged and their flow rates carefully adjusted until a single fringe covered the field as shown in Fig. 8(d). At this point the residual wedge angle of $\sim 4$ arc sec is reduced to less than 0.5 sec. of arc. The temperature differential across the face of the etalon at this point is $\sim 2^{\circ} \mathrm{C}$. Figure 8 (e) was taken 1 min later and shows a slight shift upward.

The single fringe pattern remained stable for several minutes and could be easily reset from any other steadystate pattern by careful adjustment of hot and cold water flow rates.

It appears that thermal gradients offer an excellent method of tilt angle control in SSE's.

\section{SSE as a Variable Reflector}

The reflection characteristics of a lossless FabryPerot etalon are complementary to its transmission characteristics. In a high-finesse etalon, the reflectivity is nearly unity at all frequencies except for those lying near the transmission peaks. At these resonant frequencies the reflectivity is zero. The fine temperature control of the SSE which was discussed in Sec. VII makes it practical to use the etalon as a variable reflector at any given wavelength. To demonstrate this aspect of the SSE, we used it as one of the two end reflectors that comprise the optical resonator in a $6328-\AA \mathrm{He}-\mathrm{Ne}$ laser oscillator. Thermal tuning of the etalon was then used to vary the reflectivity presented by the SSE to the laser mode. To simplify the interpretation of the results, the laser excitation was reduced to a point where only one longitudinal mode oscillated.

Figure 9(a) shows the power output from the SSE end of the laser while Curve 9 (b) shows, on the same time scale, what happens to the internal cavity energy as monitored through a high reflectivity mirror at the other end of the laser cavity. The bandwidth of the SSE passbands is about $7 \times 10^{8} \mathrm{c} / \mathrm{s}$. When a passband is tuned past the laser frequency, the output rises to a maximum at optimum coupling ${ }^{7}$ and then falls off abruptly to zero as the passband coincides with the laser oscillation frequency. At this point, the transmission losses (reduced reflectivity) exceed the amplification provided by the laser medium and the oscillation is quenched. The energy density inside the laser resonator, Fig. 9(b), is nearly a constant in the region of high etalon refiectance and drops when the passbands are approached due to the increase in external coupling.

\section{$X$. Selective Frequency Coupling with the SSE}

We have also used the SSE as a selective frequency coupler in a parametric modulation experiment. The experiment has been described previously ${ }^{8}$ so that only a short account of it is given here in order to round off the list of applications of the SSE.

An electro optic crystal (KDP in this case) was placed inside a $6328-\AA \mathrm{He}-\mathrm{Ne}$ laser and modulated by a microwave field at a frequency $f_{m}=9 \mathrm{Gc} / \mathrm{sec}$. The crystal orientation with respect to the laser polarization and propagation directions was such that only the phase of the optical field was affected by the modulation field, while the polarization direction remained unchanged. Under these circumstances the interaction is a pure parametric process, whereby a small fraction of the energy from the main laser oscillation at $f_{0}$ is transferred $^{9}$ to new frequencies at $f_{0} \pm f_{m}$. The SSE etalon was substituted for one of the end reflectors of the laser and thermally adjusted so the new frequency $f_{0}+f_{m}$ coincided with a passband of the SSE while at $f_{0}$ the reflectivity is near its maximum. The result was that most of the energy at $f_{0}+f_{m}$ was coupled out of the optical resonator while the main laser energy at $f_{0}$ was reflected by the etalon so that oscillation at $f_{0}$ could be sustained. The experimental arrangement and the relative spectral position of the laser oscillation frequency $f_{0}$, the modulation side frequencies $f_{0} \pm f_{m}$, and

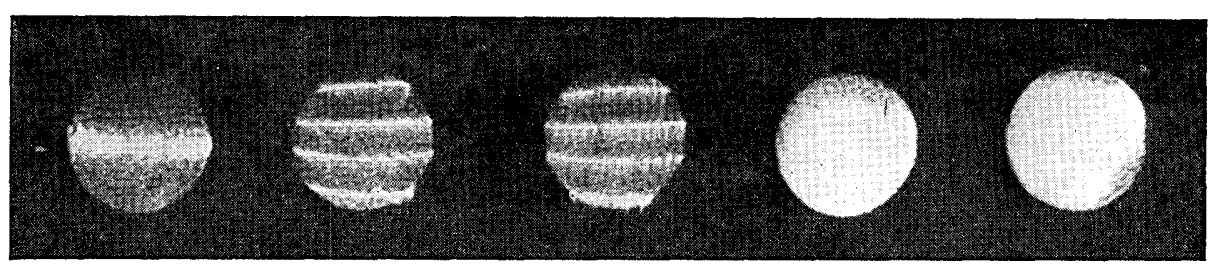

(a)

(b)

(c)

(d)

(e)

Fig. 8. Transmission pattern of solid-state etalon with thermal-gradient wedge-angle control. (a) Residual single fringe with no wedge angle correction applied. (b) Increased wedge angle caused by approximately $12^{\circ} \mathrm{C}$ temperature gradient. (c) Same fringe pattern as in (b) taken 1 min later shows a slight shift downward indicating a small average temperature change. (d) Decreased wedge angle caused by approximately $2^{\circ} \mathrm{C}$ gradient opposite in direction to that applied in (b) and (c).

(e) Same single fringe as in (d) taken 1 min later shows a slight shift upward. 


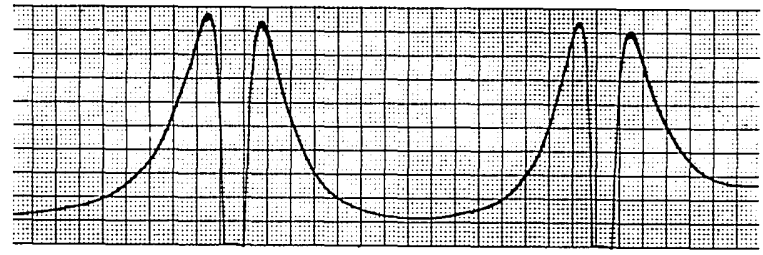

(a)

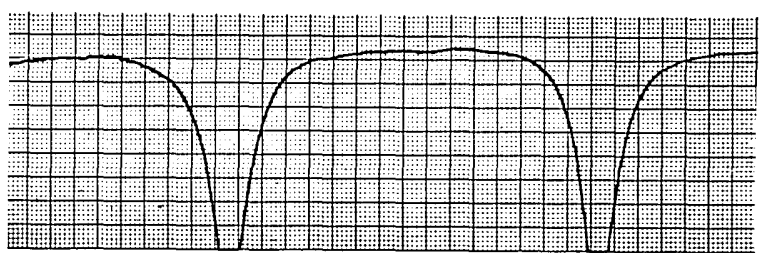

(b)

Fig. 9. Relative power output from the two ends of a $\mathrm{He}-\mathrm{Ne}$ laser controlled with a solid state etalon used as a variable reflectivity end mirror. (a) Power output from solid state etalon mirror. (b) Power output from high reflectivity mirror on the same time scale indicates what happens to the internal cavity energy.

the SSE passbands are shown in Fig. 10. The existence of the new frequency $f_{0}+f_{m}$ in the ouput beam was demonstrated by passing the (diverging) output beam through a second SSE used, conventionally, as a high resolution spectrometer. Figure 11(a) shows the output in the absence of a modulation signal or alternatively when the passband of the output SSE was tuned away from $f_{0}+f_{m}$. The family of concentric circle segments is due to light at $f_{0}$. With the modulation
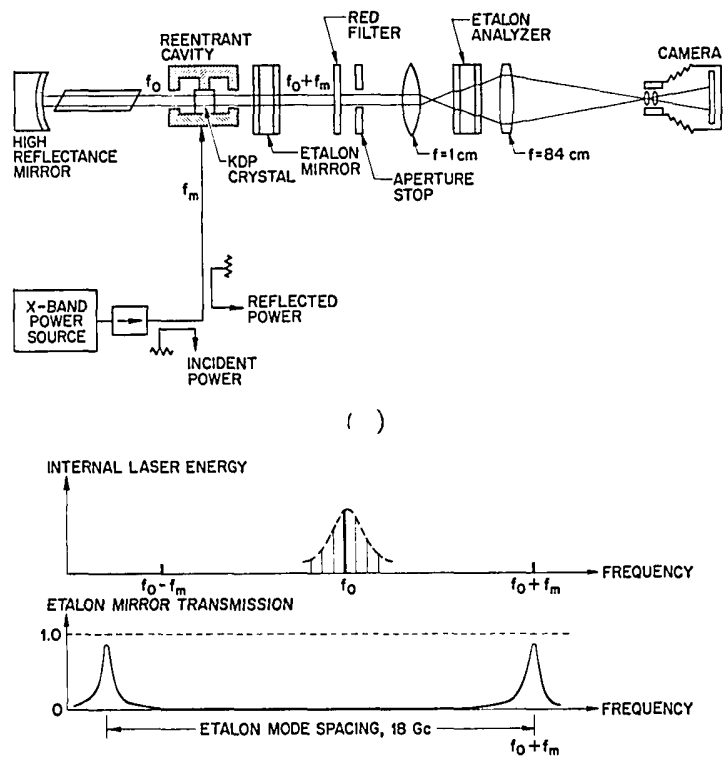

(b)

Fig. 10. (a) Diagram of the experimental set up for performing frequency translation within a laser and frequency selective output coupling. (b) The relative positions of the laser oscillation frequency $f_{0}$, the modulation sideband frequencies $f_{0} \pm f_{m}$, and the solid state etalon passbands. signal on and the etalon tuned so that one of its passbands occurs at $f_{0}+f_{m}$, the coupled light at $f_{0}+f_{m}$ gave rise to the intermediate circles seen in Fig. 11(b). We have also suggested the use of the etalon mirror for extracting a single frequency from an fm laser. ${ }^{10}$ This idea has been implemented recently by Harris and MeMurtry. ${ }^{11}$

\section{Off-Axis Transmission}

The transmission characteristics of the Fabry-Perot etalon can be studied not only by varying its optical length but also, according to Eq. (2), by changing the incidence angle $\theta$. Equation (2) or Eq. (5) predicts that

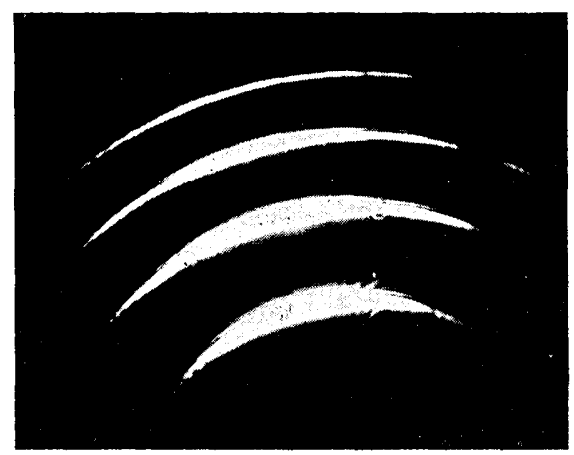

(a)

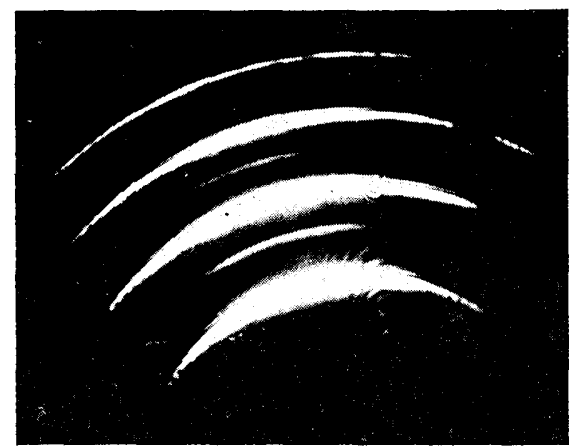

(b)

Fig. 11. Output of internally modulated laser analyzed by a Fabry-Perot interferometer with intermode spacing of $18 \mathrm{Gcsec}$ (a) Fringe system corresponding to nonmodulated oscillation at $6328 \AA$. (b) Fringe system with internal modulation at $8.9 \mathrm{Gc} \mathrm{sec}$ The new intermediate fringes indicate the presence of laser output power shifted by $8.9 \mathrm{Gc}$ sec from the laser oscillation frequency.

as $\theta$ is varied the transmission goes through a succession of peaks of equal amplitude. In practice, this is not the case. The reason is that Eq. (2) is derived assuming a beam with an infinite cross-sectional area. Under these conditions all the successive reflections from a given surface interfere with each other. This assumption is obeyed for normal incidence, $\theta=0$, if the angular spread of the incident beam is considerably smaller than the acceptance aperture $\theta_{\frac{1}{2}}=\left(\lambda_{0} / 2 n L f\right)^{\frac{1}{2}}$, which is the case in our experiment. For off-axis incidence of a finite-diameter beam, each successive reflection is accompanied by a translation $2 L \tan \theta$ of the beam across 
the reflector face. If this displacement is not a negligible faction of the beam diameter $D$, part of the interference is lost and the peak transmission is reduced. It follows from the above arguments that the peak transmission goes down with increasing $\theta$ and, secondly, that the reduction is more pronounced, all things being equal, in beams of smaller diameter. Both of these effects are clearly seen in Fig. 12. The data presented are that of the transmission (full vertical scale $=100 \%$ transmission) as a function of $\theta$ (plotted horizontally). Figures 12(a) and 12(b) were taken with $d=3.2 \mathrm{~mm}$ and $d=1.4 \mathrm{~mm}$. respectively. In neither case was the interference condition for normal incidence $\left(\theta=0^{\circ}\right)$, Eq. (1), satisfied, so that at $\theta=0^{\circ}$ there is near-zero transmission. The reduction in intensity between successive peaks is noticeably larger in the small-diameter beam, Fig. 12(b). From Fig. 12 it is clear that for small diameter beams any information obtained by scanning $\theta$, rather than $L$, could be misleading.

\section{Conclusion}

The solid state Fabry-Perot etalon has been used in conventional high resolution optical spectroscopy and in a number of new applications. These include the detection of small amounts of low-angle scattering, as a variable reflector and in laser output coupling. The dependence of the optical path length on temperature has been used for scanning the etalon and for the correction of its residual wedge angle.

The authors are indebted to Spectra-Physics Incorporated for the loan of a single-frequency laser unit.

\section{References}

1. M. Born and E. Wolf, Principles of Optics (The Macmillan Company, New York, 1964), 2nd ed.

2. C. Dufour and R. Picca, Rev. Opt. 24, 19 (1945).

3. R. Chabbal, J. Phys. Radium 19, 295 (1958).

4. V. N. Del Piano and A. F. Quesada, Appl. Opt. 4, 1386 (1965).

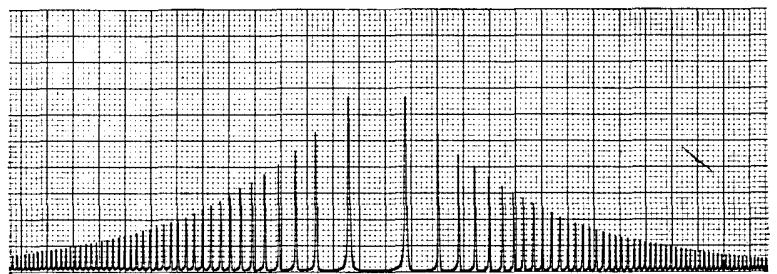

(a)

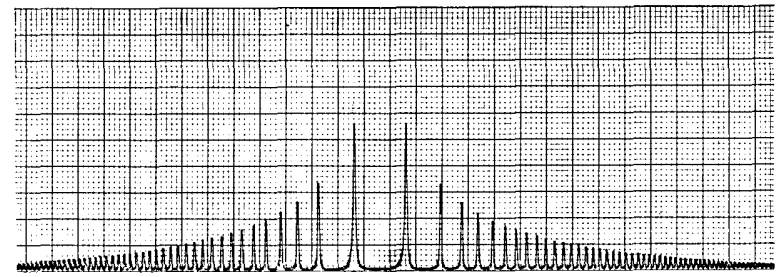

(b)

Fig. 12. The off-axis transmission characteristics of a solid-state etalon when illuminated by beams of finite size. Full vertical scale is $100 \%$ transmission. The off-axis angle is zero at the midpoint between the maxima and increases symmetrically by approximately $1^{\circ}$ per major horizontal division. The etalon length is $6 \mathrm{~mm}$. (a) Incident beam diameter $D=3.2 \mathrm{~mm}$. (b) Incident beam diameter $D=1.4 \mathrm{~mm}$.

5. H. W. Moos, G. F. Imbusch, L. F. Mollenauer, and A. L. Schawlow, Appl. Opt. 2, 817 (1963).

6. Brochure, $\mathrm{SiO}_{2}$ (Engelhard Industries, Inc., Amersil Quartz Division, Hillsdale, N.J., 1963).

7. A. Yariv, in Quantum Electronics III, P. Grivet and N. Bloembergen, Eds. (Columbia University Press, New York, 1964), Vol. 2, pp. 1055-1064.

8. D. G. Peterson and A. Yariv, Appl. Phys. Letters 5, 184 (1964).

9. A. Yariv, J. Appl. Phys. 36, 388 (1965); A. Yariv, Trans. IEEE QE-2, 30 (1966).

10. D. G. Peterson and A. Yariv, Technical Brief for Research on Single Frequency Output Gas Lasers, Lockheed Missiles \& Space Company, Palo Alto, Calif., 1965 (unpublished).

11. S. E. Harris and B. J. McMurtry, Appl. Phys. ${ }^{\ddagger}$ Letters 7, 289 (1965).

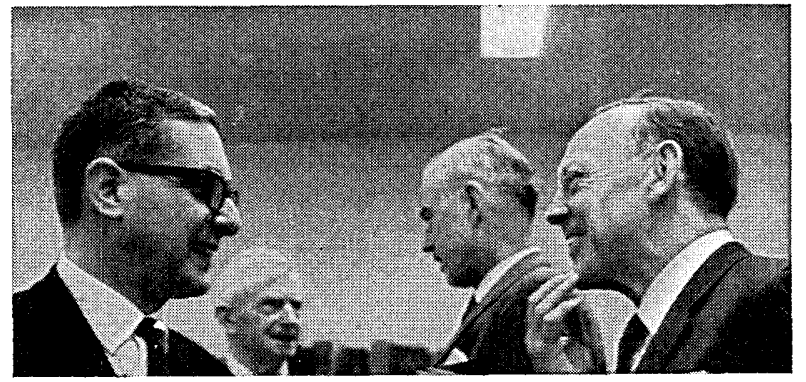

D. de Solla Price Yale (left), S. Chapman University of Alaska, Willis Lamb Yale, and C. Strutt Essex during the Rayleigh Dedication ceremonies at AFCRL in March. 


\section{The college contribution}

is a two-

way street

There are two ways to look at it.

There's the contribution the colleges make to business.

That's crucial.

Business employs about $42 \%$ of all college people. It uses their brainpower and skill in developing new products and methods. It fills management posts.

In the other direction, there's the contribution business makes to colleges.

The colleges welcome it. They need all the funds they can get. They're helping to prepare leaders for management, but the cost of education is going up.

In fact many colleges are having to turn away applicants $-100,000$ a year, says one estimate.

If business wants college talent, it must keep colleges in business. It can help finance their need for classrooms, facilities and especially teachers.

In this light, your aid-to-education program is an aid to your company.

SPECIAL TO CORPORATE OFFICERS-A NEW booklet of particular interest if your company has not yet established an aid-toeducation program. Write for:

"How to Aid Education - and Yourself" Box 36, Times Square Station New York, N. Y. 10036

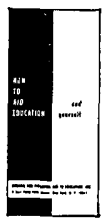

College is Business' Best Friend 